In der Rubrik „Literatur kompakt" dieser Ausgabe referieren und kommentieren diese Experten für Sie Arbeiten aus der internationalen Fachliteratur.

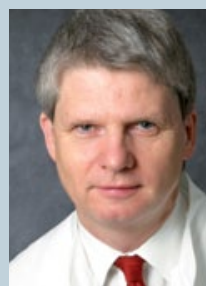

Prof. Dr. Dietrich Abeck, München

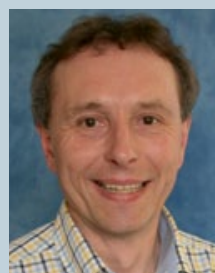

Dr. Thomas Hoppen, Koblenz

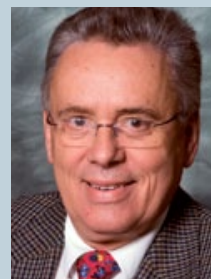

Dr. med. Hartmut Koch, Vechta

\section{Bei chronischem Husten auch an GÖR denken}

Chronischer Husten ist ein häufiges Problem, mit dem der Pädiater in der täglichen Praxis konfrontiert ist. Bis zu 20\% der Vorschulkinder und bis zu $10 \%$ der Schulkinder sind davon betroffen. Gastro-ösophagealer Reflux (GÖR) gilt als einer der häufigsten ätiologischen Faktoren, da er durch verschiedene Mechanismen in der Lage ist, Husten zu induzieren.

A ls mögliche Pathomechanismen des Hustens bei Reflux werden laryngeale Reizungen, chronische pulmonale (Mikro-)Aspirationen oder vagale Reflexe diskutiert. Da GÖR aber auch häufig ein selbstlimitierendes Phänomen ohne Symptome bei Kindern und Jugendlichen ist, besteht die Schwierigkeit, den kausalen Zusammenhang zwischen GÖR und Husten zu belegen. Symptome wie Sodbrennen, retrosternales Brennen, Brechreiz oder Bauchschmerzen können wegweisend sein; ihre Abwesenheit schließt einen pathologischen Reflux aber keineswegs aus.

Als diagnostischer Standard gilt bisher die Kombination aus Symptomen mit der Durchführung einer 24h-Langzeit-pHMetrie. Zusätzlich bieten fettbeladene Makrophagen aus dem Bronchialsekret einen diagnostischen Hinweis. Ein klarer "Goldstandard" der Diagnostik fehlt aber bisher. Zusätzlich hilfreich ist die Impedanzmessung. Diese ist in der Lage, sowohl anterograde als auch retrograde Flüssigkeitsbewegungen zu detektieren. Zusätzlich zeichnet sie neben sauren Refluxen $(\mathrm{pH}<4)$ auch leicht saure Refluxe (pH 4-7) und alkalische Refluxe auf.

Ghezzi et al. untersuchten in einer retrospektiven Studie Kinder, die zwischen 2007 und 2010 unter chronischem Husten unklarer Genese über mindestens acht Wochen litten. Diese Kinder durchliefen ein standardisiertes Untersuchungsprogramm einschließlich einer Impedanzmessung. Ausgeschlossen wurden ehemalige Frühgeborene, Kinder mit neurologischen Erkrankungen, Schluckstörungen, Anomalien der Atemwege oder des Herz-Gefäß-Systems sowie Patienten mit der Verdachtsdiagnose Asthma. Untersucht wurden insgesamt 106 Patienten, die in drei Altersgruppen unterteilt wurden: $<2$ Jahre, 2-6 Jahre und > 6 Jahre.

Von den untersuchten 106 Kindern aller Altersgruppen hatten über $70 \%$ typische Symptome, die eine GÖR vermuten ließen. Von den gesicherten Refluxereignissen waren $73,1 \%$ sauer und $26,9 \%$ schwachsauer. Alkalische Refluxe wurden nicht festgestellt. 87,7\% der untersuchten Patienten hatten mindestens eine Hustenepisode während der Refluxmessung.

Bei 78,3\% der Patienten wurde ein zeitlicher Zusammenhang zwischen nächtlichen Hustenepisoden und Refluxereignissen gefunden. Dies war bei allen drei Altersgruppen der Fall, wobei die Schulkinder signifikant mehr saure Refluxe aufwiesen als die Kleinkinder $<2$ Jahren. Auf der anderen Seite wiesen Letztere signifikant mehr schwachsaure Refluxe auf.

Insgesamt zeigte die Studie, dass saure und schwachsaure Refluxe signifikante Ursachen für chronischen Husten bei

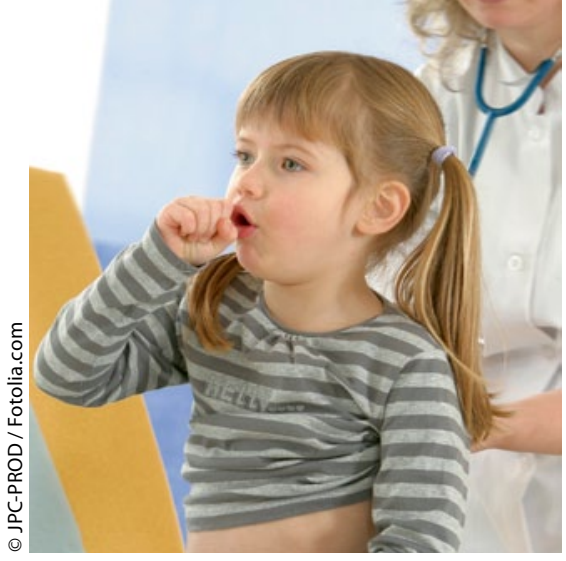

Gastro-ösophagealer Reflux ist ein häufiger Trigger bei chronischem Husten.

Kindern sind - wobei saure Refluxe insbesondere bei älteren Kindern, schwachsaure vor allem bei Kleinkindern beobachtet werden. Dies könnte auch ein Erklärungsmodell für die kürzlich veröffentlichte Cochrane-Analyse sein, dass bei Kleinkindern eine Therapie mit Protonenpumpenhemmern nicht immer wirksam ist.

Ghezzi M et al. Weakly acid gastroesophageal refluxes are frequently triggers in young children with chronic cough. Pediatr Pulmonol 2013; 48: 295-302

Kommentar: Therapierefraktärer chronischer Husten bei Kindern sollte abgeklärt werden. Hier kommt als häufige Ursache ein pathologischer GÖR in Betracht. Die basale Diagnostik umfasst die Anamnese, eine flexible Bronchoskopie mit Untersuchung auf fettbeladene Makrophagen im Bronchialsekret sowie eine 24h-Langzeit-pH-Metrie. Wenn hier kein eindeutiger saurer Reflux nachweisbar ist oder wenn die Therapie mit einem Protonenpumpenhemmer nicht erfolgreich ist, sollte sich eine Impedanzmessung anschließen - wobei Letztere mit einem großen zeitlichen und personellen Aufwand verbunden ist und auch erhebliche Materialkosten verursacht.

Dr. Thomas Spindler 